\title{
CAR/FoxP3-engineered T regulatory cells target the CNS and suppress EAE upon intranasal delivery
}

\author{
Moa Fransson ${ }^{1 \dagger}$, Elena Piras ${ }^{2 \dagger}$, Joachim Burman ${ }^{3}$, Berith Nilsson ${ }^{1}$, Magnus Essand ${ }^{1}$, BinFeng Lu ${ }^{4}$, Robert A Harris ${ }^{5}$, \\ Peetra U Magnusson ${ }^{1}$, Eva Brittebo ${ }^{2}$ and Angelica SI Loskog ${ }^{1 *}$
}

\begin{abstract}
Background: Multiple sclerosis (MS) is an autoimmune disease of the central nervous system (CNS). In the murine experimental autoimmune encephalomyelitis (EAE) model of MS, T regulatory (Treg) cell therapy has proved to be beneficial, but generation of stable CNS-targeting Tregs needs further development. Here, we propose gene engineering to achieve CNS-targeting Tregs from naïve CD4 cells and demonstrate their efficacy in the EAE model.

Methods: $\mathrm{CD}^{+} \mathrm{T}$ cells were modified utilizing a lentiviral vector system to express a chimeric antigen receptor (CAR) targeting myelin oligodendrocyte glycoprotein (MOG) in trans with the murine FoxP3 gene that drives Treg differentiation. The cells were evaluated in vitro for suppressive capacity and in C57BL/6 mice to treat EAE. Cells were administered by intranasal (i.n.) cell delivery.

Results: The engineered Tregs demonstrated suppressive capacity in vitro and could efficiently access various regions in the brain via i.n cell delivery. Clinical score 3 EAE mice were treated and the engineered Tregs suppressed ongoing encephalomyelitis as demonstrated by reduced disease symptoms as well as decreased IL-12 and IFNgamma mRNAs in brain tissue. Immunohistochemical markers for myelination (MBP) and reactive astrogliosis (GFAP) confirmed recovery in mice treated with engineered Tregs compared to controls. Symptom-free mice were rechallenged with a second EAE-inducing inoculum but remained healthy, demonstrating the sustained effect of engineered Tregs.
\end{abstract}

Conclusion: CNS-targeting Tregs delivered i.n. localized to the CNS and efficiently suppressed ongoing inflammation leading to diminished disease symptoms.

Keywords: MS, redirected cells, T regulatory cells, EAE, FoxP3, Myelin oligodendrocyte glycoprotein (MOG)

\section{Background}

Multiple sclerosis (MS) is an autoimmune disease of the central nervous system (CNS) involving autoreactive $\mathrm{T}$ cells recognizing myelin epitopes. Activated $\mathrm{T}$ cells invade the CNS, recruit peripheral mononuclear phagocytes and demyelination in the brain and spinal cord tissue, ultimately leading to impaired neuronal transmission [1]. $\mathrm{T}$ regulatory cells (Tregs) have the capacity to

\footnotetext{
*Correspondence: angelica.loskog@igp.uu.se

${ }^{\dagger}$ Equal contributors

'Department of Immunology, Genetics and Pathology, Science for Life Laboratory, Uppsala University, Rudbeck Laboratory C11, Dag

Hammarskjoldsv 20, SE-75185, Uppsala, Sweden

Full list of author information is available at the end of the article
}

regulate ongoing immune reactions and are important in the control of autoimmunity [2,3]. Tregs exert their immunosuppressive functions via secretion of inhibitory cytokines, by interfering with the metabolism of $\mathrm{T}$ cells and/or in an undetermined contact-dependent manner. Furthermore, Tregs block $\mathrm{T}$ cell activation indirectly via their interaction with antigen-presenting cells (APCs), preventing APC maturation and consequently downregulate their expression of costimulatory molecules and cytokine secretion $[4,5]$. Many studies have investigated the role of Tregs and their suppressive function in MS patients and despite some contradicting results, likely due to the multiple definitions of Treg subclasses, it has been concluded that their suppressive activities are

\section{Ciomed Central}

(C) 2012 Fransson et al.; licensee BioMed Central Ltd. This is an Open Access article distributed under the terms of the Creative Commons Attribution License (http://creativecommons.org/licenses/by/2.0), which permits unrestricted use, distribution, and reproduction in any medium, provided the original work is properly cited. 
impaired during disease progression [6-8]. MS patients may therefore benefit from Treg cell therapy to restore this insufficient immunosuppressive capacity.

It has also been demonstrated that Tregs play a critical role in the protection and recovery of the animal model of MS, experimental autoimmune encephalomyelitis (EAE). Depletion of Tregs inhibits natural recovery from EAE whereas transfer of Tregs to recipient mice reduces disease severity $[9,10]$. Transfer of antigen-specific Tregs derived from TCR transgenic mice was more effective than polyclonal Tregs in controlling murine models of both autoimmune gastritis [11] and MS [12]. However, adequate numbers of antigen-specific Tregs are difficult to achieve for adoptive transfer. A chimeric antigen receptor (CAR) was used to redirect Tregs to a desired antigen in a colitis model $[13,14]$. Cultured Tregs may change their suppressive phenotype posttreatment, and this might be detrimental for patients. For example, Xu and coworkers have reported that Tregs in the absence of TGF $\beta$ can differentiate into Th17 cells, which are considered an integral cause of autoimmune manifestations in MS [15]. Since FoxP3 is fundamental for the differentiation and maintenance of Tregs, a stable expression of FoxP3 by genetic engineering may block Treg conversion into effector cells and thereby provide a safer option for patients. In a model of arthritis, FoxP3 was coexpressed with an antigen-specific TCR to achieve multiple stable targeting Tregs [16]. By co-expressing FoxP3 with a chimeric antigen receptor (CAR) [17] targeting myelin oligodendrocyte glycoprotein (MOG) in naive $\mathrm{CD} 4^{+} \mathrm{T}$ cells we can generate sufficient numbers of stable Tregs that localize to the CNS. The role of the CAR $\alpha M O G$ receptor is to attach the Treg to the vicinity of MOG + oligodendrocytes to prevent immune attacks against these cells.

In the present study, engineered Tregs were analyzed for their suppressive function, capacity to localize to the CNS upon intranasal (i.n) or intraperitoneal (i.p) cell delivery, and for their therapeutic capacity in EAE symptomatic mice.

\section{Materials and methods}

\section{Antibody production, purification and} immunohistochemistry

Hybridoma cell line 8-18 C5 [18] was cultured in RPMI 1640 medium supplemented with $10 \%$ fetal calf serum. Antibodies were purified using protein A affinity chromatography (HiTrap MabSelect, GE Healthcare, Little Chalfont, UK) following addition of $0.5 \mathrm{M}$ trisodium citrate (Sigma-Aldrich Corp., St Louis, MO, USA) to the clarified supernatant. The column was washed with $500 \mathrm{mM}$ sodium citrate $\mathrm{pH} 8.5$ and the antibody fraction was eluted with $0.1 \mathrm{M}$ glycine (Sigma-Aldrich) at $\mathrm{pH}$ 2.7. The eluate was neutralized using Tris- $\mathrm{HCl}$ (Sigma) at
pH 8 and concentrated using a JumboSep ultrafiltration device and 10kD cutoff filter (Pall Gellman, WWR International, Stockholm). Specificity of the antibody was confirmed through Western blotting analyses of whole mouse myelin and recombinant MOG.

For immunohistochemical MOG detection sections from a naïve mouse brain were fixed in $4 \%$ paraformaldehyde and rinsed with PBS-Tween before addition of peroxidase blocking reagent (EnVision, DakoCytomation, Glostrup, Denmark). Sections were first blocked using $100 \mu \mathrm{l}$ (1:100) Fc-receptor block (Serotec, Kidlington, UK) and thereafter staining was performed with $200 \mu \mathrm{L}$ (1:50) $\alpha \mathrm{MOG}$ antibodies (8.18 C5, C. Linington) followed by $200 \mu \mathrm{l}$ (1:50) rabbit- $\alpha$-mouse Ig (EnVision, DakoCytomation) for 30 minutes each. AEC solution (DakoCytomation) was applied for colour development and sections were finally counterstained with hematoxylin prior to microscopic analysis.

\section{Chimeric antigen receptor (CAR) construct}

The CAR $\alpha$ MOG-FoxP3 vector (Figure 1A) was constructed as follows: a single chain variable fragment (scFv) was cloned from hybridoma (8.18 C5) producing anti-rat myelin oligodendrocyte glycoprotein (MOG) antibodies. The $\mathrm{scFv}$ was linked via an antibody hinge region to the transmembrane and intracellular part of a $C D 3 \zeta$ chain, which was in turn fused to an intracellular $C D 28$ domain. The murine FoxP3 gene was inserted into the construct and separated from the CAR gene by a 2A peptide (described in reference [19]). The final CAR $\alpha M O G-F o x P 3$ construct was inserted into the lentivector pRRL-CMV (kind gift from R Houeben, Leiden University Medical Center, Netherlands). Lentiviruses (Lenti-CAR $\alpha M O G-F o x p 3$ and Lenti-Mock, Lenti-GFP) were produced by co-transfecting 293FT cells with pLP1, pLP2 and pLP/VSVG (Invitrogen, Paisley, UK). Virus supernatants were harvested on days 2 and 3 and concentrated by ultracentrifugation. The amino acid sequence for the CAR $\alpha M O G$ receptor is given in Additional file 1: Figure S1.

\section{Genetic engineering of T cells}

Murine naive CD4 cells were sorted using the MACS bead system (Miltenyi, Bergisch, Germany) and prestimulated with an initial dose of $1 \mu \mathrm{g}$ of both anti-CD3 and anti-CD28 immobilized antibodies (BD Biosciences, San Diego, CA, USA) as well as IL-2 (R\&D systems Inc., Minneapolis, MN, USA) for three days prior to viral transduction, since a good viral gene transduction of $\mathrm{T}$ cells require cycling cells. $50 \mu \mathrm{l}$ of viral supernatants was added to $5 \times 10^{5}$ stimulated $\mathrm{CD}_{4}^{+} \mathrm{T}$ cells in $100 \mu \mathrm{l}$ RPMI-1640 medium supplemented with $1 \%$ sodium pyruvate, $1 \%$ nonessential amino acids, $10 \%$ fetal bovine serum, 1\% penicillin/streptomycin (all from Invitrogen, 


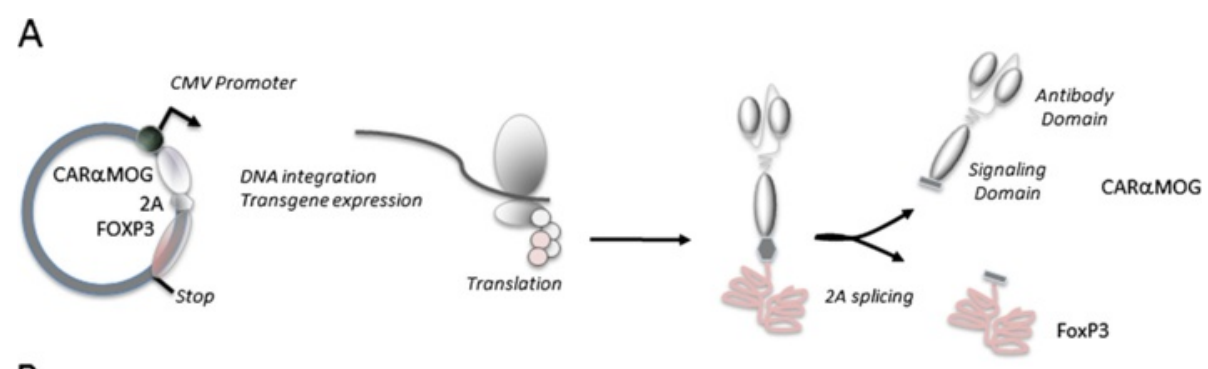

B

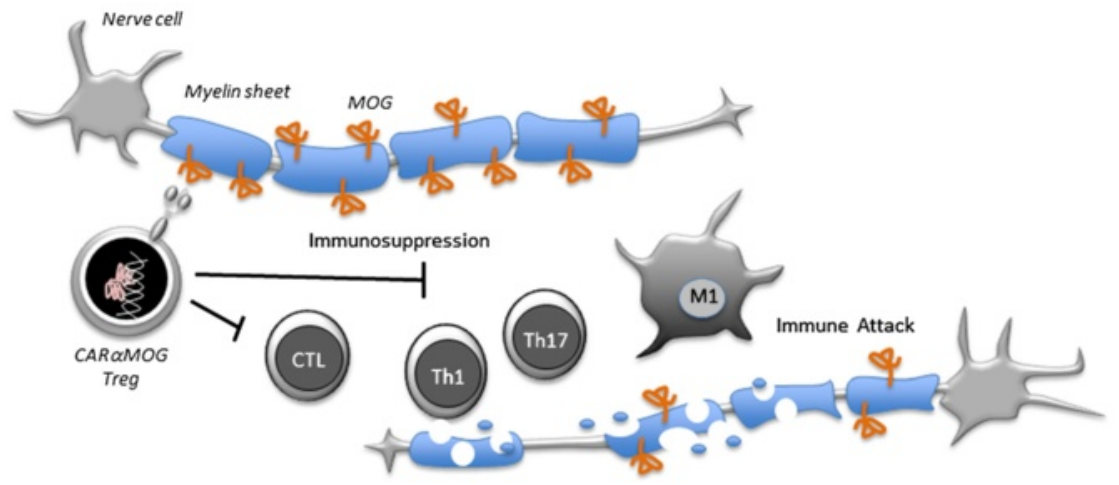

Figure 1 CNS-targeting receptor and FoxP3 translation and function. (A) The CARaMOG-FoxP3 vector contains a scFv cloned from the 8.18 C5 hybridoma. The scFv is linked via an antibody hinge region to the transmembrane and intracellular part of a CD3 zeta chain. The zeta chain is further fused to an intracellular CD28 domain. The murine FoxP3 gene was inserted into the construct after a $2 \mathrm{~A}$ peptide sequence. Upon translation, the whole expression cassette is translated into a CARFoxP3 fusion protein that is self-cleaved at the 2A site to produce the two separate proteins CARaMOG and FoxP3. (B) CARaMOG and FoxP3 is transported to the cell surface and nucleus, respectively. At the cell surface CARaMOG can bind to MOG + cells to attach the Treg to those cells and prevent immune attacks on MOG + cells such as oligodendrocytes in the CNS. FoxP3 will drive the Treg phenotype by regulating gene transcription in the nucleus.

Paisley Scotland) and $8 \mu \mathrm{g} / \mathrm{ml}$ Polybrene (Sigma-Aldrich Corp., Saint Louis, MO, USA). Cells were incubated for four hours at $37{ }^{\circ} \mathrm{C}, 5 \% \mathrm{CO}_{2}$ followed by addition of $300 \mu \mathrm{l}$ of media (as above) supplemented with 100U IL2 . The following day, media (as above) was replaced with fresh media supplemented with 80U IL-2. Cells were cultured for seven days with addition of $80 \mathrm{U}$ of IL-2 every second day. Transduction efficiency was analyzed three-to-six days post-transduction. Transduced cells were incubated for 10 minutes at $4{ }^{\circ} \mathrm{C}$ with a FITCconjugated $\mathrm{mAb}$ specific for the IgG-kappa in the $\mathrm{scFv}$ (BD Biosciences, San Diego, CA, USA), washed with PBS and resuspended in 1\% paraformaldehyde (PFA) in PBS. Samples were analyzed for surface expression of CAR or intracellular green fluorescent protein (GFP) expression using a FACScanton (BD Biosciences, San Diego, CA, USA).

\section{EAE induction and Treg cell administration}

Female C57BL/6 mice were purchased from Taconic, Lille Skensved, Denmark. Mice were housed in the Department of Animal Resources facilities at Uppsala University and used at five to eight weeks of age. Studies were approved by the regional animal ethics committee in Uppsala (C28/10). EAE was induced by subcutaneous (s.c.) immunization in both hind and front limbs with $200 \mu \mathrm{g} \mathrm{MOG}_{35-55}$ peptide emulsified in complete Freunds' adjuvant (CFA) (Difco Laboratories, Detroit, MI, USA) containing $5 \mathrm{mg} / \mathrm{ml}$ Mycobacterium tuberculosis. Pertussis toxin (100 ng i.p) (Sigma-Aldrich Corp., Saint Louis, MO, US) was given at the time of immunization and a second dose two days later. Disease severity was monitored according to the following scale: 0 , no disease; 1 , flaccid tail; 2 , hind limb weakness; 3 , hind limb paralysis; 4 , fore limb weakness; 5 , moribund. When the mean score value was 3 (usually at day 15), mice were treated using cell therapy. Cells $\left(1 \times 10^{5}\right.$ CAR or Mock-transduced Tregs diluted in $10 \mathrm{uL}$ PBS) or phosphate-buffered saline (PBS) were administered i.n. in $5 \mu \mathrm{L}$ PBS using a plastic catheter connected to a pipette (polyethylene tube, Becton Dickinson, Franklin Lakes, NJ, USA) inserted for $3 \mathrm{~mm}$ in both nasal nostrils during anesthesia (0.05 to $0.1 \mathrm{mg}$ ketalamine-xylazine mixture $/ 10$ g body weight; ketamine $50 \mathrm{mg} / \mathrm{ml}$, Pfizer AB, Sollentuna, Sweden; xylazine $20 \mathrm{mg} / \mathrm{ml}$, Bayer AG Animal Health, Business Group, Leverkusen, Germany). For i.p. cell therapy $1 \times 10^{5}$ cells (CAR or Mocktransduced Tregs) diluted in $100 \mu \mathrm{L}$ PBS were injected. Mice were sacrificed with gaseous $\mathrm{CO}_{2}$ and brains were excised and fixed either in ice-cold $4 \%$ phosphate- 
buffered formaldehyde (pH7.4) or isopentane with dryice for paraffin-embedding or frozen- sectioning, respectively. Tissues embedded in low-melting paraffin after grade scale alcohol dehydration and xylene treatment were sectioned in the sagittal plane $(4 \mu \mathrm{m})$ through the brain, mounted on gelatine-coated glass and used for immunohistochemistry.

\section{Tissue localization of engineered Treg cells in naïve mice} $1 \times 10^{4}$ GFP/CAR $\alpha$ MOG-FoxP3-engineered $\mathrm{CD} 4^{+} \mathrm{T}$ cells diluted in $5 \mu \mathrm{l}$ PBS, Mock-transduced Tregs or PBS were administered i.n in the right nostrils of naïve animals as described above. Horizontal cryosections of the brain, $(10 \mu \mathrm{m})$ were air-dried and kept at $-80{ }^{\circ} \mathrm{C}$. Tissue sections at the same level were selected following a quick staining with toluidine blue. Sections washed in cold PBS were quenched with $0.3 \% \mathrm{H}_{2} \mathrm{O}_{2}$ in methanol, blocked with $2.5 \%$ normal horse serum for one hour followed by staining with anti-GFP primary antibody (1:300) ab390 (Abcam, Cambridge, UK) overnight at $4{ }^{\circ} \mathrm{C}$. Thereafter, Alexa Fluor 488 anti-rabbit (1:200) secondary antibody was applied. Specificity controls for immunostaining included sections stained in the absence of primary antibody and staining of sections from a vehicle-treated mouse not receiving $\mathrm{GFP}^{+}$cells. For detection of DNA/nuclei sections were overlain with Vectashield Mounting Medium containing 4',6'-diamidino2-phenylindoledihydrochloride(DAPI) (Vector Laboratories, Burlingame, CA, USA). Immunofluorescence images were captured using a Leica DMRBE fluorescence microscope, a digital camera (Nikon Dxm 1200 F Nikon Corp., Tokyo, Japan) and Nikon ACT-1 version 2.62 software. All images were processed in Adobe Photoshop and Illustrator CS4, and green (GFP) and blue (DAPI) channel images were merged using Photoshop software.

\section{Immunohistochemistry for nerve damage and repair}

For myelin basic protein (MBP) and glial fibrillary acidic protein (GFAP) detection the avidin biotin complex $(\mathrm{ABC})$ method and 3,3' diaminobenzidine (DAB) as chromogen were used. Deparaffinized and rehydrated sagittal sections were rinsed with PBS and PBS-T. For GFAP antigen, demasking was performed in a microwave using $10 \mathrm{mM}$ sodium citrate buffer. Endogenous peroxidase activity was blocked with 1 to $3 \% \mathrm{H}_{2} \mathrm{O}_{2}$ in PBS-T and nonspecific background staining was blocked with 4\% BSA in PBS. Sections were incubated overnight with the primary antibodies (MBP 1:200 Abcam, Cambridge, UK; GFAP 1:400 Millipore, Billerica, MA. USA). After washing, the sections were incubated with a biotinylated secondary antibody and then with $\mathrm{ABC}$ complex (both from Vector Laboratories, Burlingame, CA, USA). Immunoreactions were visualized with DAB (SigmaAldrich Corp., St.Louis, MO, USA). Sections were counterstained with hematoxylin. Finally, the tissue sections were rinsed gradually through a graded alcohol series and finally in xylene, and mounted immediately after with Pertex (Histolab, Göteborg, Sweden). The tissue sections were analyzed using an Olympus microscope (Olympus, Tokyo, Japan) and images were captured using a digital camera as described above. Results were analyzed in a blinded mode scoring the level of staining as weak, moderate or strong. Digital images were collected at the same time using identical settings with respect to image exposure time and image compensation setting. Images were processed using Adobe Photoshop and Illustrator CS4.

\section{Treg suppression assay}

For in vitro suppression assays, $3 \times 10^{4}$ CAR $\alpha M O G-$ FoxP3 or Mock-transduced $\mathrm{CD}^{+}{ }^{+} \mathrm{T}$ cells irradiated at 25 Gy were mixed in different ratios with $\alpha \mathrm{CD} 3 / \mathrm{IL}-2$-stimulated splenocytes derived from a naïve healthy mouse in a total volume of $200 \mu \mathrm{l} /$ well. The RPMI-1640 medium was supplemented with $0.1 \%$ sodium pyruvate, $1 \%$ nonessential amino acids, $1 \%$ Hepes buffer, $1 \% \beta$ mercaptoethanol, $10 \%$ fetal bovine serum and $1 \%$ penicillin/streptomycin (all from Invitrogen, Paisley, UK). Cells were seeded in 96-well rounded-bottom tissue culture treated plates (Sarstedt, Newton, NC, USA) and incubated for 48 hours, after which $1 \mu \mathrm{Ci}$ of ${ }^{3} \mathrm{H}$-thymidine (PerkinElmer, Waltham, MA, USA) was added per well. Cells were incubated for an additional eight hours before harvest to filters. The incorporated ${ }^{3} \mathrm{H}$-thymidine was measured using a $\beta$-counter (Perkin Elmer Life Science, Turku, Finland). In some experiments, murine $2.5 \times 10^{4}$ macrophages or $2.5 \times 10^{4} \mathrm{MOG}^{+}$cells were added to cultures with CAR $\alpha M O G-F o x P 3$-transduced $\mathrm{CD} 4^{+} \mathrm{T}$ cells in a 1:1 ratio. Activated macrophages were obtained via plastic adherence of splenocytes. Monocytes were activated by $1 \mu \mathrm{g}$ lipopolysaccharide (LPS) and matured during one week in RPMI-1640 medium supplemented with $1 \%$ sodium pyruvate, $1 \%$ nonessential amino acids, $10 \%$ fetal bovine serum and $1 \%$ penicillin/ streptomycin. $\mathrm{MOG}^{+}$cells were generated via lentiviral gene transfer of murine MOG to $293 \mathrm{~T}$ cells. MOG expression was confirmed by histochemistry using $\alpha \mathrm{MOG}$ antibodies (clone 8.18 C5) as described above.

\section{Quantitative PCR}

Brain biopsies from EAE mice treated i.n. with CAR, Mock-transduced Tregs or PBS, respectively, were treated with tissue lysis buffer ATL (Qiagen, Hilden, Germany) at $60{ }^{\circ} \mathrm{C}$ for three hours followed by DNA purification using High Pure Viral Nucleic Acid kit (Roche, Basel Switzerland). cDNA was obtained using the Superscript II Reverse Transcriptase kit (Invitrogen, Paisley, UK). Quantitative-PCR was performed using the real-time 
system (iCycler, Bio-Rad Laboratories Inc., Hercules, CA, USA). The reaction was performed with SYBR green mix (BioRad). Primer pairs for $\beta$-actin were designed as follows: forward 5'-TTCCTTCCCAGAGTTCTTCCAC, reverse 5'-CCAGGATGGCCCATCGGATAAG (Cybergene $\mathrm{AB}$, Huddinge, Sweden). Primers to detect IL-12 and IFN $\gamma$ were designed as described previously $[19,20]$. In order to correct for variable amounts of DNA content between samples, all copy numbers were corrected to $\beta$-s. The mRNA copy number in $2 \mu \mathrm{L}$ cDNA was evaluated in the experiments.

\section{Statistics}

Significant differences between groups were calculated using GraphPad Software (La Jolla, CA, USA). The method for each individual calculation is stated in the Figure Legends. ${ }^{*} P<0.05,{ }^{* *} P<0.01$, ${ }^{* * * *} P<0.001$.

\section{Results}

\section{Phenotype and function of engineered Tregs}

Antibodies produced and purified from the $8.18 \mathrm{C} 5$ hybridoma were tested for cross-reactivity to murine MOG. The selected MOG antibody detected murine MOG in the brains of naive mice (Figure 2A). A scFv from the 8.18 C5 hybridoma was generated and cloned into a murine CAR receptor and was then inserted in tandem with murine FoxP3 into a lentiviral system to produce CAR $\alpha M O G-F o x P 3$ viruses. Sorted and preactivated naive $\mathrm{CD}^{+} \mathrm{T}$ cells were successfully transduced with the CAR $\alpha M O G-F o x P 3$ lentivectors (CAR Tregs). Prior to stimulation and transduction $\mathrm{CD} 4+\mathrm{T}$ cells are sorted from splenocytes. Transduced and expanded cells remain CD4 positive. Post-gene transfer the $\mathrm{scFv}$ of the CAR $\alpha \mathrm{MOG}$ receptor could be detected on the cell surface of approximately 10 to $15 \%$ of cells (Figure 2B) and the FoxP3 mRNA levels in the engineered cells were two-fold greater than that of Mock-transduced T cells (CD4 Mock) which include a population of naturally occurring Tregs (Figure 2C). Natural Tregs suppress activated $\mathrm{T}$ cells in a non-TCR restricted manner by contact dependent and independent mechanisms. CAR Tregs suppressed polyclonally stimulated T cells $(P<0.05)$ at a $1: 2$ ratio (Figure 2D) demonstrating the gained suppressive function. The role of the CAR $\alpha M O G$ receptor is to attach the Treg to the vicinity of $\mathrm{MOG}+$ oligodendrocytes to prevent immune attacks against these cells (Figure 1B). To investigate that the CAR Tregs retained their suppressive function upon binding to MOG + cells, the suppressive function of polyclonally stimulated $\mathrm{T}$ cells were analyzed in cocultures with $\mathrm{MOG}+$ cells. In Figure 2D it is demonstrated that the CAR Tregs continue to suppress $\mathrm{T}$ cell proliferation in the presence of MOG + cells. Further, activated murine macrophages may produce cytokines or other factors that block the function of the CAR Tregs. Activated macrophages are part of the MS pathology and therefore CAR Tregs were cultured with such cells to determine if CAR Tregs were still suppressive against $\mathrm{T}$ cells. In our assay CAR Tregs were still able to suppress effector $\mathrm{T}$ cell proliferation in the presence of activated macrophages (Figure 2D; $P<0.05$ ).
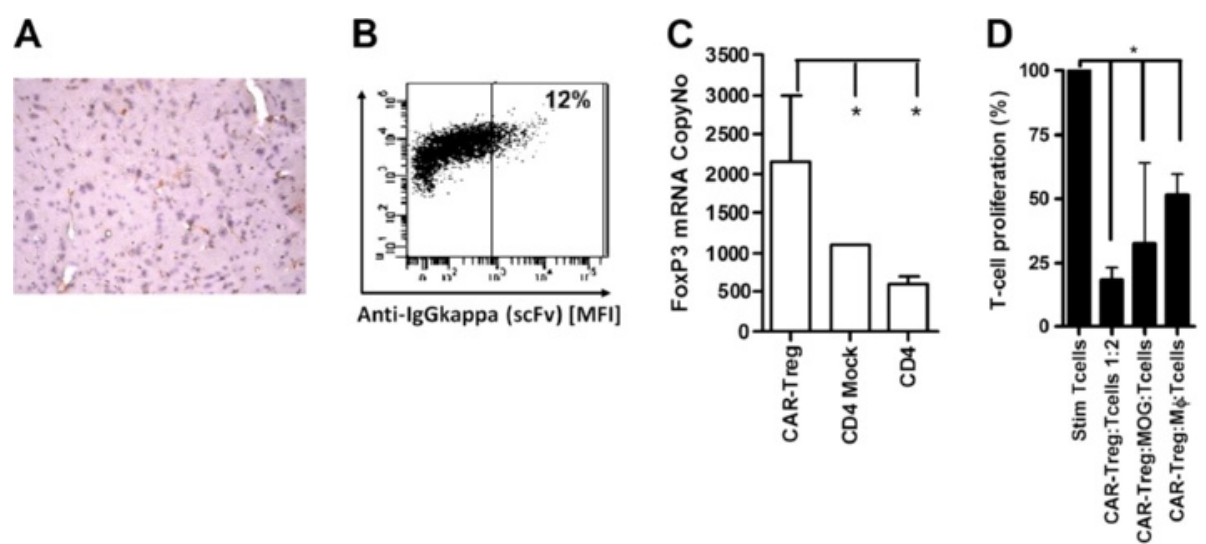

Figure 2 Engineered Tregs express CAR and FoxP3. (A) Antibodies from the 8.18 C5 hybridoma cross-reacted with murine myelin as demonstrated by immunohistochemistry (red colour expression). (B) $C D 4{ }^{+} T$ cells transduced with CARaMOG-FoxP3 lentivectors (CAR Tregs) were stained with fluorescein isothiocyanate (FITC)-conjugated antibodies and analyzed for surface expression of the scFv by flow cytometry. (C) FoxP3 copy number in transduced cells analyzed by quantitative PCR analysis. Expression of FoxP3 is significantly higher than both CD4-mock and naïve $C D 4^{+} T$ cells $(P<0,05)$ as analyzed by Mann-Whitney using GraphPad prism software. The expression experiments were repeated for every cell generation with similar result. (D) CAR Tregs were mixed with aCD3/IL-2-stimulated T cells at a1:2 ratio and analyzed for suppressive ability in a thymidine-based assay. CAR Tregs were able to suppress activated T-cells $(P<0.05)$. CAR Tregs suppressed aCD3/IL-2-stimulated T cells in the presence of activated macrophages $(P<0.05)$ or MOG-expressing cells $(P<0.05)$. The experiments were repeated and statistical differences analyzed by Mann-Whitney test using GraphPad prism software. 


\section{In vivo localization of Tregs to the brain}

CAR Tregs co-expressing GFP and CAR $\alpha M O G-F o x P 3$ were used to evaluate in vivo targeting upon i.n. cell delivery in naïve mice. The overall localization of GFP immunofluorescence is illustrated in the schematic drawing in Figure 3. The green fluorescence was mainly localized in clusters of cells in the granular layer and the external plexiform layer of the olfactory bulb (Figure 3B, C), in the lateral septal nucleus (Figure 3E), in the central medial thalamic nucleus (Figure 3F), in the ectorhinal cortex (Figure $3 \mathrm{H}$ ), in the medial genic nucleus (Figure 3I) and in the Purkinje cell layer and white matter of the cerebellum (Figure 3K, L). In addition, green immunofluorescence was observed in anterior olfactory nucleus and anterior orbital cortex (data not included). The green immunofluorescence was only observed in the soma and was preferentially present in the perinuclear part (Figure 3F, I). Although a unilateral dose of cells was given, the localization of immunofluorescence occurred both on the ipsilateral and contralateral sides of the brain. In the vehicle control animal no, or extremely weak, green background immunofluorescence could be detected (Figure 3A, D, G, J). The localization of immunofluorescence is summarized in Table 1.

\section{CNS-targeting CAR Tregs suppress active EAE}

At the peak of EAE inflammation $1 \times 10^{5}$ cells of each CAR Tregs and Mock CD4 ${ }^{+} \mathrm{T}$ cells, or PBS alone, was administered i.n. or i.p. to 10 mice per group. EAE mice responded well to cell therapy independently of administration route (Figure $4 \mathrm{~A}$ to $\mathrm{C}$ ). The EAE scores were initially reduced upon i.n delivery of either CAR Tregs or Mock $\mathrm{CD}^{+}{ }^{+} \mathrm{T}$ cells. Seven days post-treatment only the CAR Treg treatment group exhibited a continuous reduction of clinical disease symptoms and at day 25 all mice $(n=10)$ were symptom-free (Figure $4 A)$. At the same time point, only a few mice in the Mock control group $(n=5)$ that contained a normal rate of natural

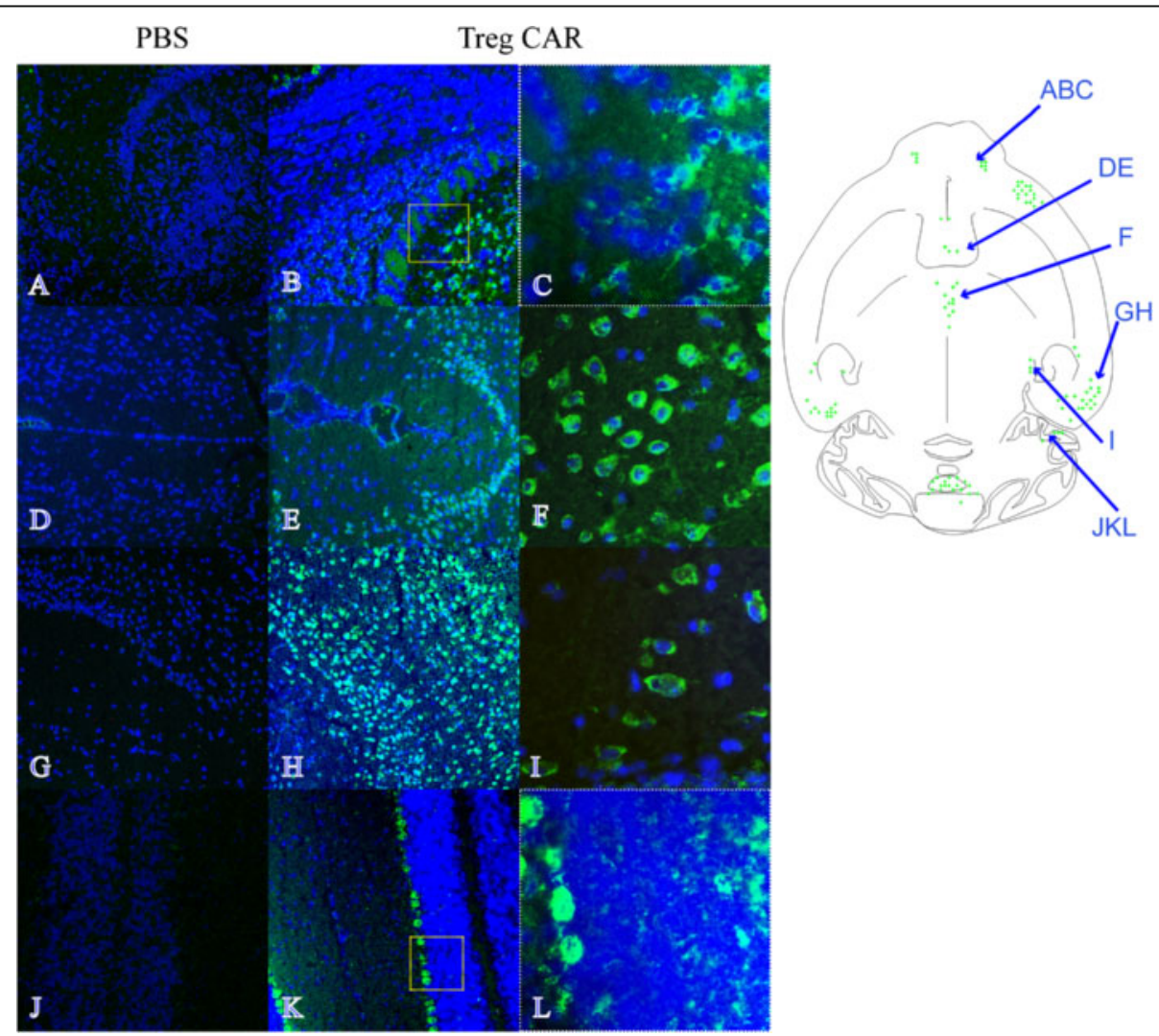

Figure 3 Engineered Tregs localize to the CNS when administered intranasally in naive mice. Treg cells were administered intranasally in the right nostril and the distribution of green fluorescent protein (GFP) in horizontal cryosections of the brain of naïve mice was studied 24 hours after the delivery. The schematic drawing describes a selective immunofluorescence in various brain regions (green spots). GFP immunofluorescence is present in the granular and to a lower extent in the external plexiform layer of the olfactory bulb (B, C), lateral septal nucleus (E), central medial thalamic nucleus $(\mathbf{F})$, ectorhinal cortex $\mathbf{( H )}$ ), medial genic nucleus $(\mathbf{I})$ and cerebellum $(\mathbf{K}, \mathbf{L})$ of a CAR Treg-treated naïve mouse. Corresponding areas showing no GFP fluorescence in a PBS-treated naïve mouse are (A, D, G, J). Enlargements of areas in olfactory bulb and cerebellum (as indicated by boxes) are depicted in $C$ and L. Detail of GFP immunofluorescence in the central medial thalamic nucleus and medial genic nucleus (F, I). Cell nuclei (blue) are stained with DAPI Original magnification 10× (A, B, D, E, G, H, J, K) and 40× (C, F, I, L). 
Table 1 Summary of GFP + cell distribution in mouse naive brain ${ }^{\mathrm{a}}$.

\begin{tabular}{ll}
\hline Tissue & GFP Immunohistochemistry \\
\hline Olfactory bulb & ++ \\
-granular layer & + \\
-external plexiform layer & - \\
-periglomerular cells & ++ \\
Anterior olfactory nucleus & ++ \\
Anterior orbital cortex & +++ \\
Lateral septal nucleus & +++ \\
Ectorhinal cortex & + \\
Central medial thalamic nucleus & ++ \\
Subgeniculates nucleus & - \\
Lateral globus pallidus & \\
Cerebellum & + \\
-molecular layer & ++ \\
-purkinje cells & + \\
-white matter &
\end{tabular}

Tregs (see Figure 2C) were classified as being healthy. The remaining mice exhibited symptoms corresponding to a clinical score of 3 . At day 30 also, the Mock control group had a good performance score and significantly differed from PBS controls $(P<0.05)$. At this time point healthy mice from each group, except the PBS treatment that did not cure mice, were re-challenged with an additional EAE-inducing inoculum using CFA and pertussis toxin. In the $\mathrm{CD}_{4}^{+}$Mock group all mice developed EAE symptoms by day two. In the CAR Treg group only one mouse developed weak EAE (score 1) (Figure 4B).

Upon examination of immunohistochemical markers for myelination (MBP) and reactive astrogliosis (GFAP), mice in the CAR Treg group exhibited confirmed recovery 15 days post-treatment (Figure 5). Reactive astrogliosis was evaluated in the olfactory bulb (Figure 5A-C), corpus callosum (Figure 5D-F), cerebellum (Figure 5G-I) and hippocampus (data not included). An increased GFAP staining was detected in both CAR Treg- and $\mathrm{CD}_{4}{ }^{+}$Mock-treated EAE mice as compared to PBStreated EAE mice. The level of staining was higher in CAR Treg-treated EAE mice as compared to $\mathrm{CD}^{+}$ Mock-treated EAE in all areas except the olfactory bulb, where the level of staining was lower (Figure 5B, C). Myelination was evaluated in the brain stem (Figure 5J-L), hippocampus (Figure 5M - O) and cerebellum (Figure 5P-R), corpus callosum and olfactory bulb (data not included). The degree of myelination, as indicated by MBP staining in PBS-treated EAE mice compared to CAR Treg-treated mice in brain stem and in cerebellum, was slightly stronger (Figure $5 \mathrm{~J}$ and $\mathrm{P}$ ), whereas staining was weak or absent in the others areas. MBP staining in the brain of $\mathrm{CD} 4^{+}$Mock-treated EAE mice was lower compared to that in CAR Treg-treated mice.

In addition to the noted markers of recovery and myelination, the levels of Th1-associated cytokines were measured by quantitative PCR analysis of tissues from the same brains. These results revealed lower levels of the T cell associated IFN $\gamma$ mRNA in mice treated with CAR Tregs compared to control brain tissue such as mice treated with PBS or Mock-transduced cells (Figure 6). IL-12, on the other hand, is only detected in PBS mice demonstrating that DC maturation may be compromised in both CAR Treg and Mock groups.

\section{Conclusions}

Tregs are developed in the thymus (natural Tregs) or in the periphery in response to cytokines such as TGF $\beta$. In autoimmunity, the patients may have genetic variations leading to reduced, or nonfunctional, Tregs. They may, as well, have deficits in other signalling pathways that affect the Treg suppression mechanisms. Severe systemic autoimmunity, such as immune polyendocrinopathy enteropathy X-linked syndrome (IPEX) shows FoxP3 mutations that block the Treg differentiation. It is difficult to dissect if natural or peripherally derived Tregs are the most important for controlling autoimmunity, but since the natural Tregs commonly have affinity for selfantigens these Tregs likely have a predominant role in blocking emerging autoimmunity $[2,3,5]$. In the current investigation, $\mathrm{CD} 4^{+} \mathrm{T}$ cells were modified utilizing a lentiviral vector system to express a chimeric antigen receptor (CAR) targeting myelin oligodendrocyte glycoprotein (MOG) in trans with the murine FoxP3 gene that drives Treg differentiation. In that sense, the gene-engineered Tregs are peripherally derived cells. However, they express FoxP3 and have a strong affinity to a self-antigen via the CAR receptor, so they are also similar to natural Tregs. The genetically engineered Tregs demonstrated suppressive capacity in vitro and reduced disease symptoms in mice with active EAE in vivo. The suppressive effects of Tregs in murine models of autoimmune pathology have provoked an interest in clinical translation. Transfer of Tregs into animals with autoimmunity provides protection but, to date, there are no records of clinical trials using adoptive transfer of Tregs in humans with autoimmune diseases. Tregs have, however, been used in the clinic to induce transplantation tolerance [21]. One problem with Tregs is sorting and expanding the population since FoxP3, the most reliable marker, is only present intranuclearly. It is also a major challenge to produce antigen-specific Tregs for cell therapy since that would require antigen-stimulation for an extended time. However, Tregs can be generated from naive $\mathrm{CD} 4^{+}$ $\mathrm{T}$ cells by gene transfer of FoxP3 [22]. Using retroviral 


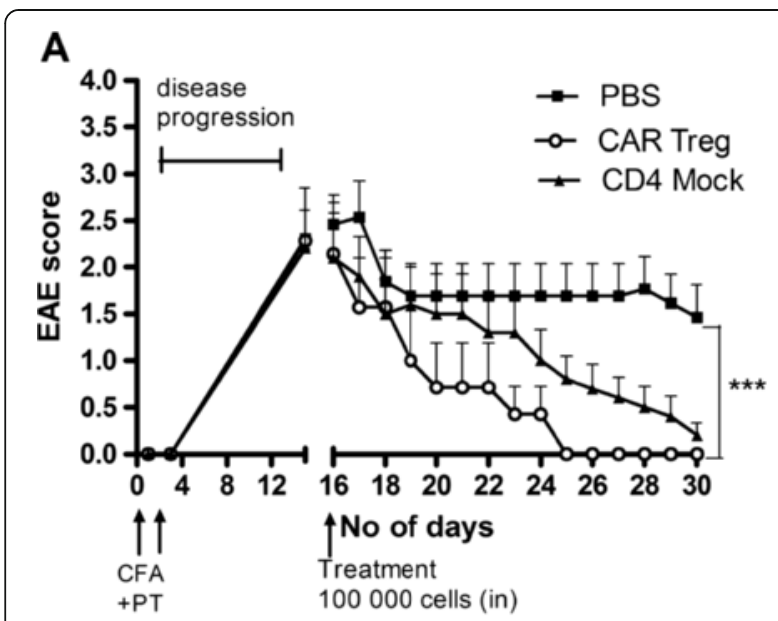

B

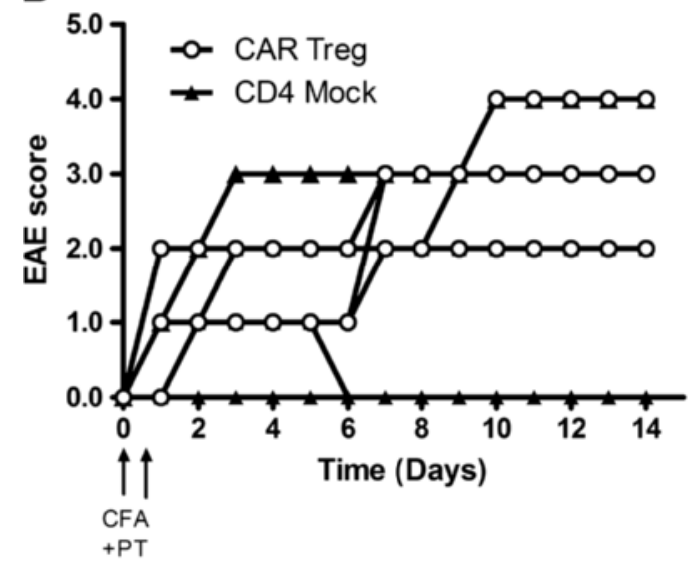

C

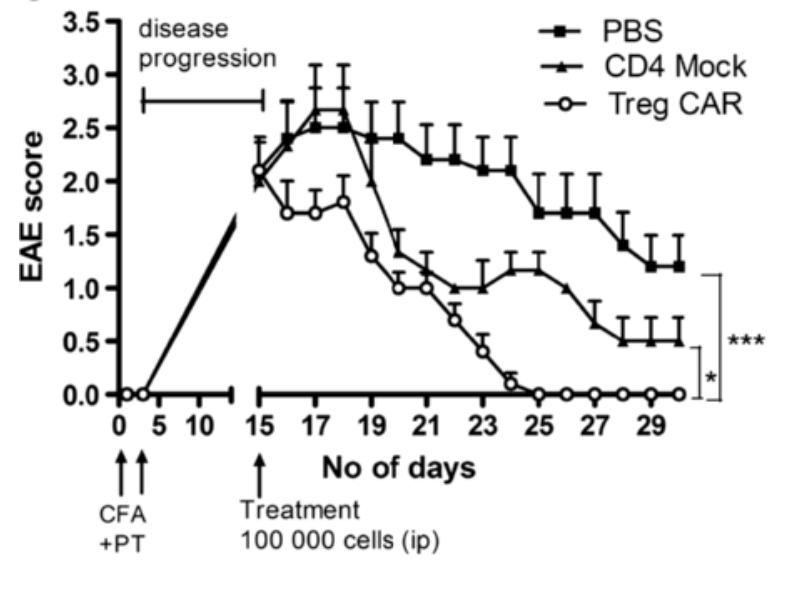

gene transfer of murine FoxP3 into $\mathrm{CD} 4^{+} \mathrm{CD} 25^{-} \mathrm{T}$ cells, Chai and coworkers generated Tregs and used them in an animal model for transplantation with promising results [23], and this was later confirmed using a lentiviral vector system [24].

Genetically engineered and cultured Tregs have been evaluated for treatment efficacy in a wide variety of
Figure 4 CNS-targeting Tregs can reduce EAE symptoms. (A)

Ten mice in three groups were given $1 \times 10^{5}$ CAR Tregs, $C D 4^{+}$Mock

$T$ cells or PBS alone by i.n. administration at the peak of EAE

inflammation (15 days post-EAE immunization) and thereafter were monitored for EAE symptoms. Ten days post cell treatment all EAE mice in the CAR Treg group were cured $(P<0.001)$. At end point (15 days post cell treatment) four out of ten EAE mice in the Mock-treated group still exhibited EAE symptoms. The experiment was repeated three times with similar results. (B) Symptom-free mice from each treatment group were given a second dose of EAE-inducing inoculum and monitored for EAE symptoms. CAR Treg-treated mice were able to resist EAE inflammation to a higher extent than $C D 4+$ Mock-treated mice $(P<0.001)$. Pooled data from six EAE mice (three/group from two separate experiments) are shown in the figure. Scores for individual mice are shown separately in the figure. (C) Ten EAE mice in three groups were administered $1 \times 10^{5}$ CAR Tregs, CD4 $4^{+}$Mock T cells or PBS alone by i.p. injection at peak of EAE inflammation (day 15). At end point (day 15 post-treatment) all mice in the CAR Treg group were cured but six out of ten mice in the Mock $C D 4^{+} \mathrm{T}$ cell group still exhibited EAE symptoms. Statistics are analyzed with Mann-Whitney test using GraphPad prism software. ${ }^{*} P<0.05,{ }^{*} P<0.01,{ }^{* *} P<0.001$.

adoptive transfer models of autoimmune diseases [9,23,25-27]. However, systemically delivered Tregs may result in recipient failure to respond to infectious disease, or they may not accumulate in sufficient amounts at the correct location. Mekala and co-workers redirected murine $\mathrm{CD} 4^{+} \mathrm{CD} 25^{+}$Tregs by using a chimeric

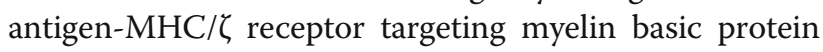
(MBP) in the EAE model. In their study MBP-specific Tregs were able to suppress EAE-inflammation [28]. Hombach and coworkers have redirected human CD4 ${ }^{+} \mathrm{CD} 25^{+}$Tregs by using retroviral transfer of a recombinant anti-CEA-immunoreceptor to target the inflamed intestine, with promising results [29]. In the present study, we have used chimeric antigen $\mathrm{T}$ cell receptors, so-called CARs, to direct the T cells to MOG present in the CNS [17]. The construct also contained murine FoxP3 to drive the transduced $\mathrm{CD}^{+}{ }^{+} \mathrm{T}$ cells toward a Treg stable phenotype.

The engineered Tregs expressed both CAR and FoxP3, and in assays testing their function they significantly decreased $\mathrm{T}$ cell proliferation even in the presence of LPS-stimulated macrophages that are thought to take part in the transformation of Tregs into Th17 effector cells due to their production of activating cytokines [30,31]. Binding to $\mathrm{MOG}^{+}$cells by their CAR did not change their suppressive function either, as indicated by co-culturing CAR Tregs, $\mathrm{MOG}^{+}$cells and stimulated $\mathrm{T}$ cells. Activated macrophages are part of the pathology of MS and the CAR T cells were challenged with such cells in the suppressive assays to exclude that they lose their function in the presence of macrophages. There was a somewhat decreased suppressive capacity noted in these groups, but it did not reach significance. 


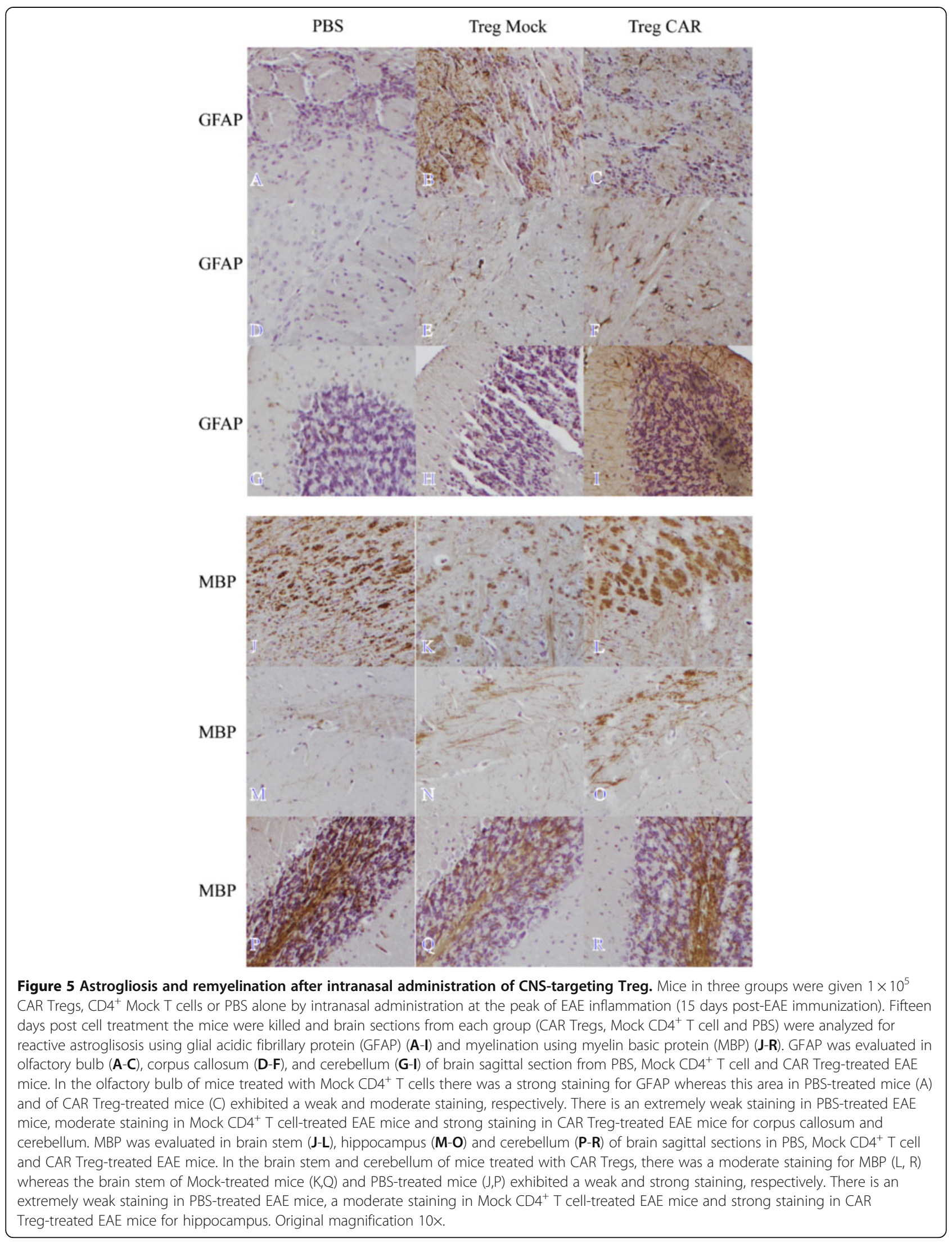




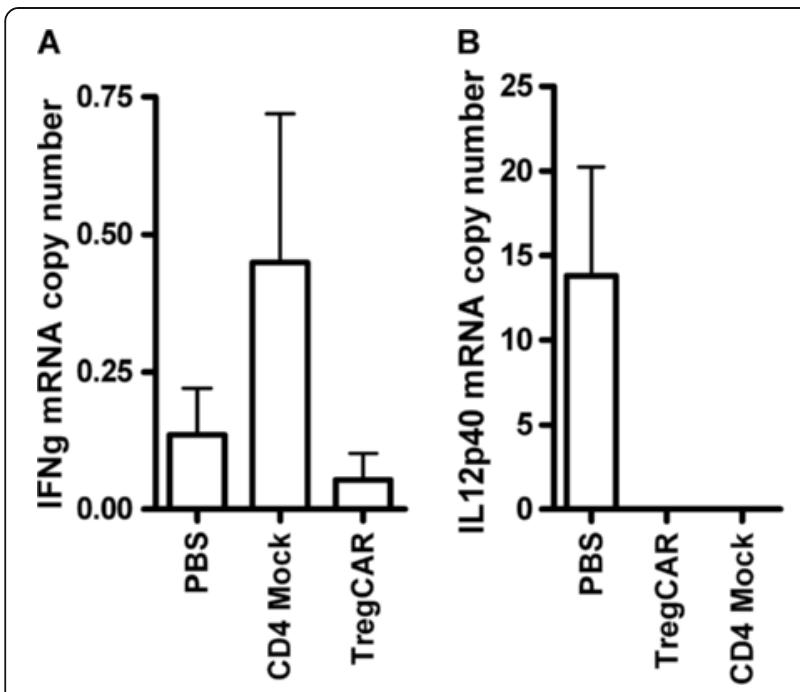

Figure 6 Decreased expression of effector cytokines in CNS-targeting CAR Treg-treated brain. Mice in three groups were given $1 \times 10^{5}$ CAR Tregs, CD4 ${ }^{+}$Mock T cells or PBS alone by i.n. administration at the peak of EAE inflammation ( 15 days post-EAE immunization). Fifteen days post cell treatment, brain biopsies from five EAE mice per group (CAR Tregs, Mock $C D 4^{+} \mathrm{T}$ cells and PBS) were analyzed for expression of effector cytokines (IL-12 and IFN $\gamma$ ) by quantitative RT-PCR. Error bars represent standard error of the mean (SEM).

GFP-expressing CAR Tregs were then used to track the immunofluorescence in the brain 24 hours after an $i$. n. cell administration. Analysis of GFP-positive immunofluorescence in the brains of naïve mice revealed clusters of fluorescent cells in various brain areas. Immunofluorescence was localized, for instance, in the olfactory bulb, orbital and ectorhinal cortex, but also in the Purkinje cells and white matter of the cerebellum. The selective GFP immunofluorescence of the Purkinje cells and other cells that are not matching was detected in the cerebellum. The selective GPF immunofluorescence of the Purkinje cells, and/or other cells that are not matching the features of Tregs, may possibly be related to a vesicular transfer and uptake of the GFP protein. Further investigations are needed to establish that the observed immunofluorescence is due to GFP-expressing CAR Tregs, fusion with other cells or cell debris that has been taken up by other cells.

A previous study has described that myelin-specific Tregs accumulate in the CNS but fail to control autoimmune inflammation [32]. This depended on the resistance of local effector $\mathrm{T}$ cells to suppression, partly due to IL-6 and TNF production. However, McGeachy and coworkers described that transfer of low numbers of $\mathrm{CD} 4{ }^{+} \mathrm{CD} 25^{+}$cells from the CNS of recovering mice before $\mathrm{EAE}$ reinduction reduces disease severity in recipients [33], demonstrating the potential of Treg therapy in EAE. Our present results clearly demonstrated that
Tregs (engineered or not) could reduce disease symptoms in mice with active EAE upon both i.n. and i.p. delivery. Hence the effector cell resistance to Treg suppression demonstrated by Korn and coworkers [34] may not occur using engineered Tregs. However, the mock-transduced $\mathrm{CD}^{+}{ }^{+} \mathrm{T}$ cells containing a mixture of natural $\mathrm{FoxP}^{+}$Tregs and naive $\mathrm{T}$ cells did not completely cure EAE. When delivered i.n. mice treated with $\mathrm{CD}^{+}$Mock $\mathrm{T}$ cells could recover from EAE nearly as well as with CNS-targeted CAR Tregs, but the effect was not optimal, since only CAR Tregs could generate mice resistant to an additional EAE challenge 30 days post-treatment. If delivered i.p. the difference between targeted and nontargeted Tregs became more evident.

Immunohistochemical evaluation of recovery (GFAP) and myelination (MBP) of axons in the brain confirmed recovery and revealed decreased damage to axons in mouse brains treated with CAR Tregs compared to control groups. Furthermore, mice treated with CAR Tregs had reduced levels of effector cytokines (IL-12 and IFN $\gamma$ ) in brain tissue compared to both mice treated with crude T cells and PBS, thus indicating the different qualities of the targeted and non-targeted Tregs in suppressing inflammation.

A problem with adoptive transfer of Tregs is the inadequate number of cells reaching the target. Cell numbers decrease during migration and the risk of therapeutic cells ending up in vital and/or reproductive organs must be taken into consideration. The olfactory pathways have been extensively investigated as potential entry for pharmaceutical drugs into the brain $[35,36]$. Recently, i.n delivery has been examined as a potential route of administration for transplantation of cells into the brain with the advantage of reducing cell doses required for therapeutic efficacy while, at the same time, decreasing systemic exposure $[32,37,38]$. In this study, we further demonstrated intranasal administration of engineered GFP + Treg cells carrying a MOG-targeting receptor can be delivered via the nostrils and access the brain.

A migration of engineered Treg in the olfactory pathways to the brain may occur via extracellular channels comprising olfactory ensheathing cells surrounding the olfactory neurons or via perivascular spaces from the nose to the brain. In addition, a migration into the general blood circulation cannot be excluded since the nasal mucosa is highly vascularized. A previous report demonstrated migration of cells from the nasal mucosa through the cribriform plate along the olfactory neural pathway into the brain and cerebrospinal fluid (CSF) following i.n. cell administration [32]. In that study, only a low number of cells were tracked up to one hour postinstillation. In the present study, we observed clusters of immunofluorescent cells in the brain 24 hours postinstillation in naïve mice. Because EAE mice treated 
once with CAR Tregs were still protected against an additional EAE rechallenge the cells may still be intact $>30$ days post initial cell therapy, but other explanations are possible. Extrapolating to the human situation, the concept presented in the current paper could facilitate administration of therapeutic cells without compromising the patient's safety or the number of cells reaching its target. In summary, i.n. cell administration of CNS-targeted CAR Tregs can be delivered to the brain using i.n. cell administration and efficiently hamper the disease symptoms as well as protect against an additional EAE challenge. The novel findings in this report warrant clinical translation.

\section{Additional file}

Additional file 1: Figure S1. The amino acid sequence of the expressed CAR targeting MOG is shown in the figure.

\section{Competing interests}

The authors declare that they have no competing interests with the contents in this paper. However, Dr. Loskog is the CEO of Lokon Pharma AB, an advisor of NEXTTOBE $A B$, and has a royalty agreement with Alligator Bioscience $\mathrm{AB}$.

\section{Acknowledgements}

This study was supported by grants to Assoc. Prof. Loskog from the Swedish Research Council and the Medical Faculty at Uppsala University, to Dr. Fransson from the Göransson-Sandviken fund, and to Prof. Essand from the Marcus and Marianne Wallenberg Foundation. The authors thank Raili Engdahl for technical assistance during the animal experiments.

\section{Author details}

'Department of Immunology, Genetics and Pathology, Science for Life Laboratory, Uppsala University, Rudbeck Laboratory C11, Dag Hammarskjoldsv 20, SE-75185, Uppsala, Sweden. ${ }^{2}$ Department of Pharmaceutical Biosciences, Uppsala University, BMC, Husarg 3, SE-75124, Uppsala, Sweden. ${ }^{3}$ Department of Neuroscience, Uppsala University, Uppsala University Hospital Entr 70, SE-75185, Uppsala, Sweden. ${ }^{4}$ Department of Immunology, University of Pittsburgh, 320 East North Avenue, Pittsburgh, PA 15212, USA. ${ }^{5}$ Applied Immunology, Department of Clinical Neurosciences, Karolinska Institutet, Center for Molecular Medicine, Karolinska Hospital at Solna, Solna, Sweden.

\section{Authors' contributions}

$M F, E P, E B$, and $A L$ designed research and wrote the paper. MF, EP, JB, RH performed experiments. BN, ME, BL, RH and AL designed separate protocols and provided the necessary scientific input to execute the project. All authors read and approved the final manuscript.

Received: 19 December 2011 Accepted: 30 May 2012 Published: 30 May 2012

\section{References}

1. Martin R, McFarland HF: Immunological aspects of experimental allergic encephalomyelitis and multiple sclerosis. Crit Rev Clin Lab Sci 1995, 32:121-182.

2. Maloy KJ, Powrie F: Regulatory T cells in the control of immune pathology. Nat Immunol 2001, 2:816-822.

3. Coutinho A, Hori S, Carvalho T, Caramalho I, Demengeot J: Regulatory T cells: the physiology of autoreactivity in dominant tolerance and "quality control" of immune responses. Immunol Rev 2001, 182:89-98.

4. Ziegler SF: FOXP3: of mice and men. Annu Rev Immunol 2006, 24:209-226.

5. Sakaguchi S, Sakaguchi N: Regulatory T cells in immunologic selftolerance and autoimmune disease. Int Rev Immunol 2005, 24:211-226.
6. Fransson M, Burmna J, Lindqvist C, Atterby C, Fagius J, Loskog A: T regulatory cells lacking CD25 are increased in MS during relapse. Autoimmunity 2010, 43:590-597.

7. Venken K, Hellings $\mathrm{N}$, Thewissen M, Somers V, Hensen K, Rummens JL, Medaer R, Hupperts R, Stinissen P: Compromised CD4+ CD25(high) regulatory T-cell function in patients with relapsing-remitting multiple sclerosis is correlated with a reduced frequency of FOXP3-positive cells and reduced FOXP3 expression at the single-cell level. Immunology 2008, 123:79-89.

8. Haas J, Hug A, Viehöver A, Fritzsching B, Falk CS, Filser A, Vetter T, Milkova L, Korporal M, Fritz B, Storch-Hagenlocher B, Krammer PH, Suri-Payer E, Wildermann $B$ : Reduced suppressive effect of $C D 4+C D 25$ high regulatory $T$ cells on the T cell immune response against myelin oligodendrocyte glycoprotein in patients with multiple sclerosis. Eur J Immunol 2005, 35:3343-3352

9. Kohm AP, Carpentier PA, Anger HA, Miller SD: Cutting edge: CD4 + CD25+ regulatory $T$ cells suppress antigen-specific autoreactive immune responses and central nervous system inflammation during active experimental autoimmune encephalomyelitis. J Immunol 2002, 169:4712-4716.

10. Zhang X, Koldzic DN, Izikson L, Reddy J, Nazareno RF, Sakaguchi S, Kuchroo $V K$, Weiner HL: IL-10 is involved in the suppression of experimental autoimmune encephalomyelitis by $\mathrm{CD} 25+\mathrm{CD} 4+$ regulatory T cells. Int Immunol 2004, 16:249-256.

11. DiPaolo RJ, Brinster C, Davidson TS, Andersson J, Glass D, Shevach EM: Autoantigen-specific TGFbeta-induced Foxp3+ regulatory T cells prevent autoimmunity by inhibiting dendritic cells from activating autoreactive $T$ cells. J Immunol 2007, 179:4685-4693.

12. Stephens LA, Malpass KH, Anderton SM: Curing CNS autoimmune disease with myelin-reactive Foxp3+ Treg. Eur J Immunol 2009, 39:1108-1117.

13. Elinav E, Adam N, Waks T, Eshhar Z: Amelioration of colitis by genetically engineered murine regulatory $T$ cells redirected by antigen-specific chimeric receptor. Gastroenterology 2009, 136:1721-1731.

14. Elinav E, Waks T, Eshhar Z: Redirection of regulatory T cells with predetermined specificity for the treatment of experimental colitis in mice. Gastroenterology 2008, 134:2014-2024.

15. Xu L, Kitani A, Fuss I, Strober W: Cutting edge: regulatory T cells induce CD4 + CD25-Foxp3- T cells or are self-induced to become Th17 cells in the absence of exogenous TGF-beta. J Immunol 2007, 178:6725-6729.

16. Wright GP, Notley CC, Xue SA, Bendle GM, Holler A, Schumacher TN, Ehrenstein MR, Stauss HJ: Adoptive therapy with redirected primary regulatory T cells results in antigen-specific suppression of arthritis. Proc Natl Acad Sci USA 2009, 106:19078-19083.

17. Pule M, Finney H, Lawson A: Artificial T-cell receptors. Cytotherapy 2003, 5:211-226.

18. Breithaupt C, Schubart A, Zander H, Skerra A, Huber R, Linington C, Jacob U: Structural insights into the antigenicity of myelin oligodendrocyte glycoprotein. Proc Natl Acad Sci USA 2003, 100:9446-9451.

19. Loskog A, Dzojic H, Vikman S, Ninalga C, Essand M, Korsgren O, Tötterman TH: Adenovirus CD40 ligand gene therapy counteracts immune escape mechanisms in the tumor Microenvironment. J Immunol 2004, 172:7200-7205.

20. Loskog A, Ninalga C, Hedlund T, Alimohammadi M, Malmström PU, Tötterman TH: Optimization of the MB49 mouse bladder cancer model for adenoviral gene therapy. Lab Anim 2005, 39:384-393.

21. Trzonkowski P, Bieniaszewska M, Juschinska J, Dobyszuk A, Krzystyniak A Marek N, Mysliwska J, Hellmann A: First-in-man clinical results of the treatment of patients with graft versus host disease with human ex vivo expanded CD4 + CD25 + CD127- T regulatory cells. Clin Immunol 2009, 133:22-26.

22. Hori S, Nomura T, Sakaguchi S: Control of regulatory T cell development by the transcription factor Foxp3. Science 2003, 299:1057-1061.

23. Chai JG, Xue SA, Coe D, Addey C, Bartok I, Scott D, Simpson E, Stauss HJ, Hori S, Sakaguchi S, Dyson J: Regulatory T cells, derived from naive CD4 + CD25- T cells by in vitro Foxp3 gene transfer, can induce transplantation tolerance. Transplantation 2005, 79:1310-1316.

24. Szymczak AL, Workman CJ, Wang Y, Vignali KM, Dilioglou S, Vanin EF, Vignali DA: Correction of multi-gene deficiency in vivo using a single 'self-cleaving' 2A peptide-based retroviral vector. Nat Biotechnol 2004, 22:589-594. 
25. Cao J, Chen C, Zeng L, Li L, Li Z, Xu K: Engineered regulatory T cells prevent graft-versus-host disese while sparing the graft-versus-leukemia effect after bone marrow transplantation. Leuk Res 2010, 34:1374-1382.

26. Wraith DC, Nicolson KS, Whitley NT: Regulatory CD4+ T cells and the control of autoimmune disease. Curr Opin Immunol 2004, 16(6):695-701.

27. Tang Q, Henriksen KJ, Bi M, Finger EB, Szot G, Ye J, Masteller EL, McDevitt H, Bonyhadi M, Bluestone JA: In vitro-expanded antigen-specific regulatory $\mathrm{T}$ cells suppress autoimmune diabetes. J Exp Med 2004, 199:1455-1465.

28. Mekala DJ, Geiger TL: Immunotherapy of autoimmune encephalomyelitis with redirected CD4 + CD25+ T lymphocytes. Blood 2005, 105:2090-2092.

29. Hombach AA, Kofler D, Rappl G, Abken H: Redirecting human $\mathrm{CD} 4+\mathrm{CD} 25+$ regulatory T cells from the peripheral blood with pre-defined target specificity. Gene Ther 2009, 16:1088-1096.

30. Valmori D, Raffin C, Raimbaud I, Ayyoub M: Human RORgt + TH17 cells preferentially differentiate from naive FOXP3+ Treg in the presence of lineage-specific polarizing factors. Proc Natl Acad Sci USA 2010, 107:19402-19407.

31. Ziegler SF, Buckner JH: FOXP3 and the regulation of Treg/Th17 differentiation. Microbes Infect 2009, 11:594-598.

32. Danielyan L, Schafer R, von Ameln-Mayerhofer A, Buadze M, Geisler J, Klopfer T, Burkhardt U, Proksch B, Verleysdonk S, Ayturan M, Buniatian GH, Gleiter CH, Frey WH 2nd: Intranasal delivery of cells to the brain. Eur I Cell Biol 2009, 88:315-324.

33. McGeachy MJ, Stephens LA, Anderton SM: Natural recovery and protection from autoimmune encephalomyelitis: contribution of $\mathrm{CD} 4+\mathrm{CD} 25+$ regulatory cells with the central nervous system. J Immunol 2005, 175:3025-3032.

34. Korn T, Reddy J, Gao W, Bettelli E, Awasthi A, Petersen TR, Bäckström BT, Sobel RA, Wucherpfennig KW, Strom TB, Oukka M, Kuchroo VK: Myelinspecific regulatory $T$ cells accumulate in the CNS but fail to control autoimmune inflammation. Nat Med 2007, 13:423-431.

35. Westin U, Piras E, Jansson B, Bergström U, Dahlin M, Brittebo E, Björk E: Transfer of morphine along the olfactory pathway to the central nervous system after nasal administration to rodents. Eur J Pharm Sci 2005, 24:565-573.

36. Illum $\mathrm{L}$ : Transport of drugs from the nasal cavity to the central nervous system. Eur J Pharm Sci 2000, 11:1-18.

37. Jiang $Y, Z h u J, X u G, L i u X$ : Intranasal delivery of stem cells to the brain. Expert Opin Drug Deliv 2011, 8:623-632.

38. van Velthoven CT, Kavelaars A, van Bel F, Heijnen CJ: Nasal administration of stem cells: a promising novel route to treat neonatal ischemic brain damage. Pediatr Res 2010, 68:419-422.

doi:10.1186/1742-2094-9-112

Cite this article as: Fransson et al:: CAR/FoxP3-engineered T regulatory cells target the CNS and suppress EAE upon intranasal delivery. Journa of Neuroinflammation 2012 9:112.

\section{Submit your next manuscript to BioMed Central and take full advantage of:}

- Convenient online submission

- Thorough peer review

- No space constraints or color figure charges

- Immediate publication on acceptance

- Inclusion in PubMed, CAS, Scopus and Google Scholar

- Research which is freely available for redistribution

Submit your manuscript at www.biomedcentral.com/submit
C Biomed Central 